\title{
Chronic abdominal pain in a patient on chronic peritoneal dialysis: question
}

\author{
Olivera Marsenic • Bernard Kaplan • \\ Kevin E. C. Meyers
}

Received: 12 March 2008/Revised: 26 April 2008 /Accepted: 27 May 2008 /Published online: 9 July 2008

(C) IPNA 2008

$\begin{array}{ll}\text { Abbreviations: } \\ \text { PD } & \text { peritoneal dialysis } \\ \text { FSGS } & \text { focal segmental glomerulosclerosis } \\ \text { UF } & \text { ultrafiltration } \\ \text { CCPD } & \text { continuous-cycler peritoneal dialysis } \\ \text { HD } & \text { hemodialysis } \\ \text { CT } & \text { computed tomography }\end{array}$

Case summary

A 19-year-old woman on chronic peritoneal dialysis (PD) presented with abdominal pain in March 2007. Focal segmental glomerulosclerosis (FSGS) was initially diagnosed at age 7 years and progressed to end-stage renal disease (ESRD). PD was started at 10 years of age and was complicated by two episodes of peritonitis. At 12 years of age, she received a renal allograft from her father. The posttransplantation course was complicated by FSGS

The answer to this question can be found at http://dx.doi.org/10.1007/ s00467-008-0925-8.

O. Marsenic $\cdot$ B. Kaplan $\cdot$ K. E. C. Meyers

Division of Nephrology,

The Children's Hospital of Philadelphia

and The University of Pennsylvania,

Pennsylvania, USA

K. E. C. Meyers $(\square)$

34th Street and Civic Center Boulevard,

Philadelphia, PA 19104, USA

e-mail: meyersk@email.chop.edu recurrence, endocarditis, rejection episodes, and lymphoma. Twenty-four months after transplant, the graft was removed and PD was restarted. Over the next 5 years, PD was complicated by persistent and progressive difficulty with ultrafiltration (UF) and inadequate solute removal with maximized continuous-cycler peritoneal dialysis (CCPD). The patient was unwilling to accept regular hemodialysis (HD), but a fistula was created and dialysis clearance was improved by adding a weekly HD session. Acute pancreatitis was diagnosed in November 2006, with left upper quadrant abdominal pain and elevated serum amylase and lipase. An abdominal computed tomography (CT) scan showed diffuse thickening of bowel loops. Conservative management resulted in symptom resolution and normalized serum amylase and lipase concentrations. In March 2007, she developed protracted abdominal pain associated with nausea, vomiting, and loose stools for 3 weeks and $2.5 \mathrm{~kg}$ weight loss in 2 weeks. Abdominal pain was band like, worse on sitting up, improved with PD fills, and worsened with PD drains. On physical examination, the abdomen was soft, there were no palpable masses or organomegaly, and bowel sounds were normal.

\section{Questions}

1. What is the differential diagnosis of abdominal pain in this patient on chronic PD?

2. What diagnostic tests would be performed?

3. What is the management of this problem? 